\title{
Modification of CFCM in The Presence of Heavy AWGN for Bayesian Blind Channel Equalizer
}

\author{
Changkyu Kim \\ Dept. of Information \& Communication Eng. \\ Dongeui University \\ Pusan, Korea 614-714
}

\author{
Soowhan Han \\ Dept. of Multimedia Engineering \\ Dongeui University \\ Pusan, Korea 614-714
}

\begin{abstract}
In this paper, the modification of conditional Fuzzy C-Means (CFCM) aimed at estimation of unknown desired channel states is accomplished for Bayesian blind channel equalizer under the presence of heavy additive Gaussian noise (AWGN). For the modification of CFCM to search the optimal channel states of a heavy noise-corrupted communication channel, a Gaussian weighted partition matrix, along with the Bayesian likelihood fitness function and the conditional constraint of ordinary CFCM, is developed and exploited. In the experiments, binary signals are generated at random and transmitted through both types of linear and nonlinear channels which are corrupted with various degrees of AWGN, and the modified CFCM estimates the channel states of those unknown channels. The simulation results, including the comparison with the previously developed algorithm exploiting the ordinary CFCM, demonstrate the effectiveness of proposed modification in terms of accuracy and speed, especially under the presence of heavy AWGN. Therefore, the proposed modification can possibly constitute a search algorithm of optimal channel states for Bayesian blind channel equalizer in severe noise-corrupted communication environments.
\end{abstract}

Keywords-Gaussian Partition Matrix; Conditional Fuzzy CMeans; Channel States; Bayesian Blind Equalizer

\section{INTRODUCTION}

Channel equalization is a major issue in digital communications, because a channel is easily affected by intersymbol-interference (ISI) with both linear and nonlinear distortions in the presence of AWGN. The task of channel equalization is to minimize those distortions to recover the transmitted sequence. In general, there exist two kinds of equalizers in digital communication systems: data aided (trained) equalizers and blind equalizers. For trained equalizers, a reference signal is required, increasing the bandwidth. However, in blind equalizations, the original transmitted message is recovered only from the received sequence that is corrupted by noise without any training sequence or a priori knowledge of the channel. As a result, the use of blind equalizers is preferred in high-speed communication systems to reduce ISI without increasing overhead costs [1][2]. Because of inherent simplicity, most available blind equalization algorithms focus on linear channels that are often inadequate for modeling channels which exhibit nontrivial nonlinearities [3]. In practical world, the equalization of nonlinear is often required such as in high power amplifiers as well as in high-density magnetic and optical storage channels. Therefore, the blind equalization

This study is supported by Dongeui University Research Grant 2013AA142 method handled in this paper must be dealt with both linear and nonlinear channels, which is independent of the type of channel structure.

Traditionally, channel equalization has been considered equivalent to inverse filtering. The optimal solution, based on maximum likelihood sequence estimation (MLSE) [4], has a complexity that grows exponentially with the dimension of the channel impulsive response (Viterbi algorithm). Alternatively, several nonlinear detection procedures have been proposed to address this problem with varying degrees of success, such as multi-layered perceptrons (MLPs) [5], radial basis function networks (RBFNs) [6], recurrent RBFNs [7], self-organizing feature maps (SOFMs) [8][9], wavelet neural networks [10], kernel Adeline (KA) [11], support vector machines (SVMs) [12] and Genetic Algorithms[13][14]. Such structures usually outperform linear equalizers, especially when non-minimum phase channels are encountered. They can also compensate for nonlinearities in the channel. However, they still suffer from the relatively high computational cost such as the iterative reweighted quadratic procedure of SV in [12]. The simplex Genetic Algorithm (GA) in [13] estimates the optimal channel output states instead of estimating the channel parameters in a direct manner. The desired channel states of an unknown channel were constructed from these estimated channel output states, and placed at the center of RBF equalizer. With this approach, the complex modeling of the nonlinear channel can be avoided and the method works well within a simple single input single output (SISO) communication environment. Additionally, this kind of approach can be applied to a linear channel as well, because it does not estimate the channel parameters but the channel output states directly, which is not dependent on the type of the channel structure. However, the GA based algorithms may visibly suffer from their poor convergence properties. Recently, to overcome this weakness, Fuzzy C-Means (FCM), one of the representative clustering algorithms which exhibits shorter processing time than the GA-based methods, has been modified and applied, and the faster convergence speed along with the reliable estimation accuracy in search of the optimal channel output states has been achieved [15][16]. Especially, the algorithm based on CFCM clearly outperforms the GA and FCM approaches in terms of speed and accuracy [16]. The CFCM was first introduced in [17], and successfully applied to channel equalization problem [16][18]. The conditioning aspect of CFCM, which describes a level of involvement of incoming input pattern in the constructed clusters, influences the clustering mechanism and improves the estimation accuracy 
of an unknown channel states for blind channel equalization. However, in the presence of heavy AWGN that often arises in a high speed communication channel, the estimation accuracy of CFCM presented in [16] needs the higher level of reliability, even though it is superior to other FCM or GArelated algorithms. This leads to the consideration of the modification of CFCM clustering mechanism, which makes it more robust to the heavy noise. In this study, the modification is accomplished by using a Gaussian weighted partition matrix during the clustering procedure of CFCM. The use of Gaussian weights for a partition matrix instead of ordinary Euclidean distance measuring can help to search the correct channel states of an unknown channel, because the received sequence under the presence of AWGN is a scattered random process having conditional Gaussian density functions centered at each of the desired channel states. More details on this modification of CFCM are explained in Section 5. Before that, an optimal Bayesian equalizer for a linear/nonlinear channel is introduced in the next section and the reconfiguration procedure of desired channel states with channel output states is discussed in Section 3. In Section 4, the fitness function for the proposed CFCM is derived. This study is an extension of previous work [16] and thus the similarity of the structure of Section 2 and 3 can be found in [16]. Finally, the simulation results including some comparative studies with early work [16] and conclusions are provided in Section 6 and 7, respectively.

\section{OPTIMAL BAYESIAN EQUALIZER FOR A LINEAR/NONLINEAR CHANNEL}

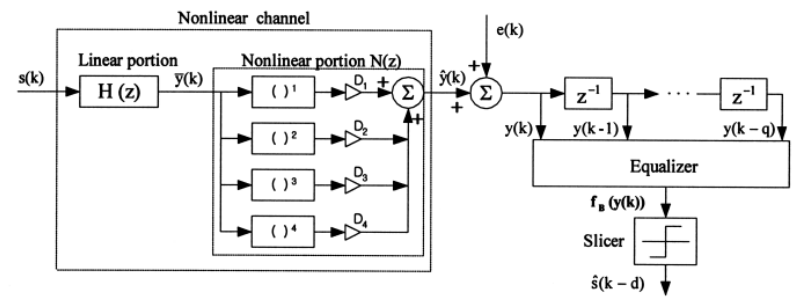

Fig. 1. Channel equalization with ISI

A general channel equalization system with ISI is illustrated in Fig. 1. The digital information symbol $s(k)$, which is assumed to be an equiprobable and independent binary sequence taking values from a two-valued set $\{ \pm 1\}$, is transmitted through a nonlinear dispersive channel. Here the nonlinear channel is composed of a linear part with transfer function $H(z)$, whose output $\bar{y}(k)$ described by (1), and a nonlinear component $N(z)$, whose output $\hat{y}(k)$ governed by (2).

$$
\begin{gathered}
\bar{y}(k)=\sum_{i=0}^{p} h(i) s(k-i) \\
\hat{y}(k)=D_{I} \bar{y}(k)+D_{2} \bar{y}(k)^{2}+D_{3} \bar{y}(k)^{3}+D_{4} \bar{y}(k)^{4}
\end{gathered}
$$

In (1) and (2), $p$ is the channel order and $D_{i}$ stands for the coefficient of the $i^{\text {th }}$ nonlinear term which is possibly caused by nonlinearities associated with nonlinear devices used in the transmitter and the receiver. For a linear channel model, the nonlinear terms are set to $D_{1}=1, D_{2}=0, D_{3}=0$ and $D_{4}=0$. The noise-free observation vector, $\hat{\boldsymbol{y}}(\boldsymbol{k})$ expressed by (3), is referred to as the desired channel states, and for a specific equalizer order denoted by $q$, there exist $M=2^{p+q+1}$ different patterns.

$$
\hat{\boldsymbol{y}}(\boldsymbol{k})=[\hat{y}(k), \hat{y}(k-1), \cdots, \hat{y}(k-q)]
$$

These $M$ desired channel states can be partitioned into two sets, $\boldsymbol{Y}_{q, d}^{+1}$ and $\boldsymbol{Y}_{q, d}^{-1}$, as shown in (4) and (5), depending on the value of $s(k-d)$, where $d$ is the required time delay.

$$
\begin{aligned}
& \boldsymbol{Y}_{q, d}^{+l}=\{\hat{\boldsymbol{y}}(\boldsymbol{k}) \mid s(k-d)=+1\} \\
& \boldsymbol{Y}_{q, \boldsymbol{d}}^{-l}=\{\hat{\boldsymbol{y}}(\boldsymbol{k}) \mid s(k-d)=-1\}
\end{aligned}
$$

Finally, the desired channel states, $\hat{\boldsymbol{y}}(\boldsymbol{k})$, are corrupted by the AWGN, $\boldsymbol{e}(\boldsymbol{k})$, and thus the channel observation vector (input of equalizer) $\boldsymbol{y}(\boldsymbol{k})$ can be described as

$$
\boldsymbol{y}(\boldsymbol{k})=\hat{\boldsymbol{y}}(\boldsymbol{k})+\boldsymbol{e}(\boldsymbol{k})
$$

The task of the equalizer is to produce the estimated sample $\hat{s}(k-d)$ which has the same value as the transmitted symbol $s(k-d)$, based on the noise-corrupted observation vector, $\boldsymbol{y}(\boldsymbol{k})$. Because of the AWGN, the observation vector, $\boldsymbol{y}(\boldsymbol{k})$, is a random process having conditional Gaussian density functions centered at each of the desired channel states, $\hat{\boldsymbol{y}}(\boldsymbol{k})$. The determination of the value of $s(k-d)$ becomes a decision problem. The optimal symbol-by-symbol spaced equalizer decision function is provided by the maximum a-posteriori probability criteria and is called Bayesian equalizer. The decision function for Bayesian equalizer [19] can be represented as follows,

$$
\begin{aligned}
f_{\boldsymbol{B}}(\boldsymbol{y}(\boldsymbol{k}))=\sum_{i=1}^{n_{s}^{+1}} \exp \left(-\left\|\boldsymbol{y}(\boldsymbol{k})-\boldsymbol{y}_{i}^{+1}\right\|^{2} / 2 \sigma_{e}^{2}\right) \\
\quad-\sum_{i=1}^{n_{s}^{-1}} \exp \left(-\left\|\boldsymbol{y}(\boldsymbol{k})-\boldsymbol{y}_{i}^{-1}\right\|^{2} / 2 \sigma_{e}^{2}\right) \\
\hat{s}(k-d)=\operatorname{sgn}\left(f_{\boldsymbol{B}}(\boldsymbol{y}(\boldsymbol{k}))\right)= \begin{cases}+1, & f_{\boldsymbol{B}}(\boldsymbol{y}(\boldsymbol{k})) \geq 0 \\
-1, & f_{\boldsymbol{B}}(\boldsymbol{y}(\boldsymbol{k}))<0\end{cases}
\end{aligned}
$$

where $\boldsymbol{y}_{i}^{+l}$ and $\boldsymbol{y}_{i}^{-l}$ are the desired channel states belonging to sets $\boldsymbol{Y}_{q, d}^{+l}$ and $\boldsymbol{Y}_{q, d}^{-1}$, respectively, and their number of elements in these sets are denoted by $n_{s}^{+1}$ and $n_{s}^{-1}$. Furthermore $\sigma_{e}^{2}$ is the noise variance. From (7) and (8), the evaluation of desired channel states is essential for the optimal Bayesian equalizer, and the performance of Bayesian blind equalizer highly depends on the correct estimation of the desired channel states, $\boldsymbol{y}_{i}^{+l}$ and $\boldsymbol{y}_{i}^{-1}$, only from the noise-corrupted observation vector, $\boldsymbol{y}(\boldsymbol{k})$. In this study, the modification of CFCM with Gaussian weighted partition matrix is presented to search the optimal states of an unknown channel under the presence of heavy AWGN. After the estimation of the desired channel states, the equalization for the reconstruction of the transmitted symbols is straightforward with (7) and (8).

\section{RECONFIGURATION OF DESIRED CHANNEL STATES WITH CHANNEL OUTPUT STATES}

The knowledge of the desired channel states, $\boldsymbol{y}_{i}^{+1}$ and $\boldsymbol{y}_{i}^{-1}$, is essential for the Bayesian equalizer. If the channel order is 
taken as $p=1$ with transfer function $H(z)=0.5+z^{-1}$, the equalizer order $q$ is equal to 1 , the time delay $d$ is also set to 1 , and the nonlinear portion is described by $D_{1}=1, D_{2}=0.0, D_{3}=-0.9, D_{4}=0.0$ (see Fig. 1), then the eight different desired channel states $\left(2^{p+q+1}=8\right)$ may be observed at the receiver in a noise-free case. The input sequences, the desired channel states and the output of the equalizer for this channel are shown in Table 1. From this table, it can be seen that the values of the desired channel states $[\hat{y}(k), \hat{y}(k-1)]$ are composed of the elements of the scalar channel states called "channel output states" , $\left\{a_{1}, a_{2}, a_{3}, a_{4}\right\}$, where for this particular channel they have $a_{1}=-1.5375, a_{2}=-0.3875$, $a_{3}=0.3875$ and $a_{4}=1.5375$. The only difference between the desired channel states and channel output states is that the first are vectors while the latter are scalars. The length of this scalar dataset, $\tilde{n}$, is determined by the channel order, $p$, such as $2^{p+1}=4$, which is independent of the equalizer order. As shown in Table 1 , the desired channel states for $\boldsymbol{Y}_{l, l}^{+1}$ (positive states) and $\boldsymbol{Y}_{l, l}^{-1}$ (negative states) are $\left(a_{1}, a_{1}\right),\left(a_{1}, a_{2}\right),\left(a_{3}, a_{1}\right)$, $\left(a_{3}, a_{2}\right)$ and $\left(a_{2}, a_{3}\right),\left(a_{2}, a_{4}\right),\left(a_{4}, a_{3}\right),\left(a_{4}, a_{4}\right)$, respectively. A change in the decision delay only changes some of the positive states to negative and the equal number of the negative states to positive. Additionally, it can be applied for a linear model as well, where nonlinear terms of channel, $D_{2}, D_{3}$, and $D_{4}$, are equal to zero. In case of the linear model, the elements of data set $\left\{a_{1}, a_{2}, a_{3}, a_{4}\right\}$ become $1.5,-0.5,0.5$ and -1.5 , respectively, and are shown in Table 1 by parentheses. The desired channel states of these nonlinear and linear models are illustrated in Fig. 2. This relationship of desired channel states and channel output states is always valid for the channel that has a one-to- one mapping between the channel inputs and outputs [13] and is successfully used in [14]-[16]. Additionally, it can be easily extended with a higher channel order such as $p=2$, which is evaluated in the experimental section. If the channel order $p$ is 2 with $H(z)=0.3482+0.8704 z^{-1}+0.3482 z^{-2}$, there exist the sixteen desired channel states $\left(2^{p+q+1}=16\right)$ composed of the eight channel output states ( $\left.\tilde{n}=2^{p+1}=8, a_{1}, a_{2}, a_{3}, \cdots, a_{8}\right)$. The desired channel states, $\left(a_{1}, a_{1}\right),\left(a_{1}, a_{2}\right),\left(a_{2}, a_{3}\right),\left(a_{2}, a_{4}\right),\left(a_{5}, a_{1}\right)$, $\left(a_{5}, a_{2}\right),\left(a_{6}, a_{3}\right),\left(a_{6}, a_{4}\right)$, belong to $\boldsymbol{Y}_{1,1}^{+1}$, and $\left(a_{3}, a_{5}\right),\left(a_{3}, a_{6}\right),\left(a_{4}, a_{7}\right)$, $\left(a_{4}, a_{8}\right),\left(a_{7}, a_{5}\right),\left(a_{7}, a_{6}\right),\left(a_{8}, a_{7}\right),\left(a_{8}, a_{8}\right)$ belong to $\boldsymbol{Y}_{l, 1}^{-1}$, where $a_{1}, a_{2}, a_{3}, \cdots, a_{8}$ are 2.0578, 1.0219, -0.1679, -0.7189, 1.0219, $0.1801,-0.7189$ and -1.0758 , respectively. This channel can be found in [16] as well. As shown in Table 1, the desired channel states for both types of linear and nonlinear can be constructed with the channel output states if channel order, $p$, is assumed to be known, and thus the main problem of blind equalization moves its focus onto the determination of the optimal channel output states only from the received patterns.

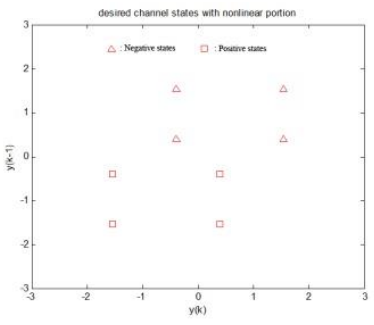

(a) for the nonlinear channel

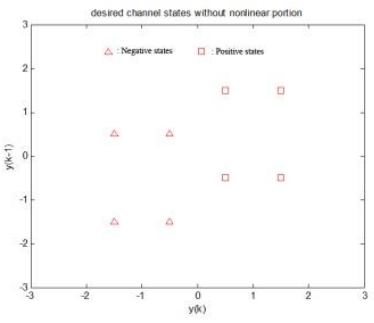

(b) for the linear channel
Fig. 2. Desired channel states (noise-free) for the nonlinear and linear channels shown in Table 1 (positive( $\square$ ) and negative $(\triangle)$ states).

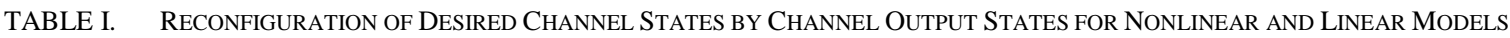

\begin{tabular}{|c|c|c|c|c|c|c|}
\hline \multicolumn{7}{|c|}{$\begin{array}{c}\text { Nonlinear channel with } H(z)=0.5+1.0 z^{-1}, D_{1}=1, D_{2}=0.0, D_{3}=-0.9, D_{4}=0.0 \text {, and } d=1 \\
\text { Linear channel with } H(z)=0.5+1.0 z^{-1}, D_{1}=1, D_{2}=0, D_{3}=0, D_{4}=0 \text {, and } d=1\end{array}$} \\
\hline \multirow{2}{*}{\multicolumn{3}{|c|}{$\begin{array}{c}\text { Input sequences } \\
s(k) s(k-1) s(k-2)\end{array}$}} & \multicolumn{3}{|c|}{ Desired channel states } & \multirow{3}{*}{$\begin{array}{c}\text { Output of equalizer } \\
\hat{s}(k-1) \\
1 \\
\end{array}$} \\
\hline & & & $\begin{array}{cc}\hat{y}(k) \quad \hat{y}(k-1) \\
\text { ( ) used for the lineal model }\end{array}$ & $\begin{array}{l}\text { By char } \\
\text { states, }\{a\end{array}$ & $\begin{array}{l}\text { loutput } \\
\left.a_{2}, a_{3}, a_{4}\right\}\end{array}$ & \\
\hline 1 & 1 & 1 & $-1.5375(1.5)-1.5375(1.5)$ & $\left(a_{1}, a_{1}\right)$ & \multirow{4}{*}{$\begin{array}{c}\text { Positive } \\
\text { channel } \\
\text { states, } \\
\boldsymbol{Y}_{1,1}^{+l}\end{array}$} & \\
\hline 1 & 1 & -1 & $-1.5375(1.5)-0.3875(-0.5)$ & $\left(a_{1}, a_{2}\right)$ & & 1 \\
\hline-1 & 1 & 1 & $0.3875(0.5)-1.5375(1.5)$ & $\left(a_{3}, a_{1}\right)$ & & 1 \\
\hline-1 & 1 & -1 & $0.3875(0.5)-0.3875(-0.5)$ & $\left(a_{3}, a_{2}\right)$ & & 1 \\
\hline 1 & -1 & 1 & $-0.3875(-0.5) \quad 0.3875(0.5)$ & $\left(a_{2}, a_{3}\right)$ & \multirow{4}{*}{$\begin{array}{c}\text { Negative } \\
\text { channel } \\
\text { states, } \\
\boldsymbol{Y}_{l, l}^{-l}\end{array}$} & -1 \\
\hline 1 & -1 & -1 & $-0.3875(-0.5) \quad 1.5375(-1.5)$ & $\left(a_{2}, a_{4}\right)$ & & -1 \\
\hline-1 & -1 & 1 & $1.5375(-1.5) \quad 0.3875(0.5)$ & $\left(a_{4}, a_{3}\right)$ & & -1 \\
\hline-1 & -1 & -1 & $1.5375(-1.5) \quad 1.5375(-1.5)$ & $\left(a_{4}, a_{4}\right)$ & & -1 \\
\hline
\end{tabular}

\section{FitNESS FUNCTION FOR OPTIMAL CHANNEL STATES}

In order to find the optimal channel states, the use of the Bayesian likelihood $(B L)$ [20] is considered. Since the Bayesian decision variable is a probability density function (pdf) variable, similar to the conventional likelihood, the $B L$ can be defined by (9).

$$
B L=\prod_{k=0}^{L-1} \max \left(f_{B}^{+1}(k), f_{B}^{-1}(k)\right)
$$

$$
\text { where } f_{B}^{+l}(k)=\sum_{i=1}^{n_{s}^{+1}} \exp \left(-\left\|\boldsymbol{y}(\boldsymbol{k})-\boldsymbol{y}_{\boldsymbol{i}}^{+1}\right\|^{2} / 2 \sigma_{e}^{2}\right), f_{B}^{-1}(k)=\sum_{i=1}^{n_{s}^{-1}} \exp
$$
$\left(-\left\|\boldsymbol{y}(\boldsymbol{k})-\boldsymbol{y}_{\boldsymbol{i}}^{-1}\right\|^{2} / 2 \sigma_{e}^{2}\right)$ and $L$ is the length of the received sequences. By evaluating the Bayesian likelihood, the optimal dataset of desired channel states which always corresponds to the maximum Bayesian likelihood would be found [13]. For this reason, the $B L$ has been widely used as a fitness function $(F F)$ in the previously developed search algorithms based on 
GA or Fuzzy Clustering [13]-[16], and it is utilized as a fitness function for our modification of CFCM as well. Being more specific, the fitness function is taken as the logarithm of the $B L$, that is

$$
F F=\sum_{k=0}^{L-1} \log \left(\max \left(f_{B}^{+1}(k), f_{B}^{-1}(k)\right)\right)
$$

Because of the characteristics of $F F$ illustrated in [13] and [16], it cannot be easily solved by conventional gradient-based methods. On the other hand, the mathematical relation between $F F$ and channel states cannot be formulated without the knowledge of channel structure [13]. Furthermore, it is too complex to be formulated even if the channel structure is known. Therefore in this paper, to search the optimal channel states which produce the maximum $F F$, under the presence of heavy AWGN, a modification of CFCM with Gaussian weighted partition matrix is developed.

\section{MOdifiCATION OF CFCM WITH GaUSSIAN WEIGHT}

The conditional fuzzy clustering method was reported by W. Pedrycz in [17] and successfully applied to channel equalization problem [16][18]. In [17], the conditioning aspect of the clustering mechanism is introduced by taking into consideration the conditioning variable assuming values, $f_{l}, f_{2}, \ldots, f_{k}$, on the corresponding patterns. This conditioning aspect, which describes a level of involvement of incoming input pattern in the constructed clusters, influences the clustering mechanism and improves the estimation accuracy of an unknown channel states for blind channel equalization [16]. Using the conditioning variables, $f_{1}, f_{2}, \ldots, f_{k}$, makes it possible to apply the different weights to each of received patterns, which depend on their distances to the constructed clusters. To be more specific, the closer the received pattern to the clusters, the higher weight is attached and consequently more influential it becomes in the clustering process. For example, if $f_{i}=0$, the $i^{\text {th }}$ received pattern is regarded as meaningless in the clustering procedure and the calculations of the resulting prototypes are not affected by this element. Subsequently, the calculations of the partition matrix $\boldsymbol{U}$ in fuzzy clustering procedure do not take this into consideration. On the other hand, the pattern for which $f_{i}=1$ contributes to the clustering process to the highest extent. This can be accomplished by the partition matrix $\boldsymbol{U}$ in CFCM derived as follows

$$
\begin{gathered}
U_{i k}^{(m+1)}=\frac{f_{k}}{\sum_{l=1}^{n_{s}}\left(\frac{\left\|\boldsymbol{y}(\boldsymbol{k})-\boldsymbol{y}_{i}^{(\boldsymbol{m})}\right\|}{\left\|\boldsymbol{y}(\boldsymbol{k})-\boldsymbol{y}_{l}^{(\boldsymbol{m})}\right\|}\right)^{2}} \\
\boldsymbol{y}_{i}^{(m+1)}=\frac{\sum_{k=0}^{L-1}\left(U_{i k}^{(m+1)}\right)^{2} \boldsymbol{y}(\boldsymbol{k})}{\sum_{k=0}^{L-1}\left(U_{i k}^{(m+1)}\right)^{2}}
\end{gathered}
$$

where $\boldsymbol{y}_{i}^{(m+1)}$ is the $i^{\text {th }}$ estimated center set at the $(m+1)^{\text {th }}$ iteration and $i=1,2,3, \ldots, 8$ for the channels in Table 1 because of $n_{s}=8$ (total number of desired channel states). By the same way, the range of $i$ for the channel in Table 2 is 1 to 16 . Here in (11), the conditional constraint $f_{k}$ should contain the distance information of each of received patterns, and it has a high value if the corresponding pattern is closely located at the estimated center. The CFCM in [16] utilizes each component of $B L$ for the received patterns shown in (9) as the conditional constraint $f_{k}$ after normalization, because it contains the distance information for each of received patterns. For an example, if a received pattern is located near the optimal desired channel states, $\boldsymbol{y}_{i}^{+l}$ or $\boldsymbol{y}_{\boldsymbol{i}}^{-1}$, this pattern produces a higher value of $f_{B}^{+l}(k)$ or $f_{B}^{-l}(k)$ in (9) and consequently it becomes more influential in the clustering process by (11). In other words, the closer the received pattern to the optimal channel states, the higher conditional constraint is applied. Because of the use of these conditioning variables, the performance of CFCM is relatively superior to those of the existing GA and FCM based approaches in terms of speed and accuracy. More details of the CFCM clustering algorithm for blind channel equalizations are described in [16]. The conditional constraint $f_{k}$ of the CFCM represents the Gaussian probability value of each of received patterns because it depends on the $B L$ in (9). However, the partition matrix $\boldsymbol{U}$ in (11) is still updated based on Euclidean distance measure. Because of AWGN, the received vector, $\boldsymbol{y}(\boldsymbol{k})$, is scattered with a conditional Gaussian probability density centered at each of the desired channel states. Therefore, for the calculation of partition matrix $\boldsymbol{U}$ during the clustering procedure, the Gaussian probability of each of received patterns should be involved instead of the Euclidean distance measuring. The Gaussian weighted partition matrix $\boldsymbol{U}_{\boldsymbol{G}}$, where Euclidean distance is replaced with Gaussian probability, is described by (13) and a new center set $\boldsymbol{y}_{\boldsymbol{i}}$ is sequentially derived by (14).

$$
\begin{aligned}
U_{i k}^{(m+1)} & =\frac{f_{k} \cdot \exp \left(-\left\|\boldsymbol{y}(\boldsymbol{k})-\boldsymbol{y}_{i}^{(m)}\right\|^{2} / 2 \sigma_{e}^{2}\right)}{\sum_{l=1}^{n_{s}} \exp \left(-\left\|\boldsymbol{y}(\boldsymbol{k})-\boldsymbol{y}_{l}^{(m)}\right\|^{2} / 2 \sigma_{e}^{2}\right)} \\
\boldsymbol{y}_{i}^{(m+1)} & =\sum_{k=0}^{L-1} U_{i k}^{(m+1)} \boldsymbol{y}(\boldsymbol{k})
\end{aligned}
$$

The effectiveness of the proposed Gaussian weighted partition matrix $\boldsymbol{U}_{\boldsymbol{G}}$ under a heavy noise environment is demonstrated in Fig. 3. It shows the values of the conditional constraint $f_{k}$ after 10 epochs of clustering procedure with the partition matrix $\boldsymbol{U}$ in (11) and $\boldsymbol{U}_{\boldsymbol{G}}$ in (13) for the nonlinear channel in Table 1. The optimal centers of this channel are illustrated in Fig. 2(a). The received patterns under OdB SNR are shown in Fig. 3(a), and the value of conditional constraint for each of those patterns is displayed by gray-colors (from 0 (white) to 1(black)) in Fig. 3(b). For both cases, the noisecorrupted patterns, which are scattered and located far away from the optimal centers, have relatively very low constraint values (close to "0" indicated by bright color in Fig. 3(b)). On the other hand, the received patterns located near the optimal channel states are more weighted by the conditional constraint $f_{k}$ (close to "1", black color in Fig. 3(b)) and generate higher contributions to the clustering procedure. However, the 
received patterns with the high values of $f_{k}$ in Fig. 3(c)(e)_left are more widely spread than the patterns in Fig. 3(c)(e)_right. In other words, it is observed that, in Fig. 3(c)(e)_right, the high constraint values are assigned only for the received patterns which are more densely located near the optimal centers. It means that, by the clustering procedure with the partition matrix $\boldsymbol{U}_{G}$, the closer located patterns near the optimal states have the relatively higher values of $f_{k}$ than the values of $f_{k}$ by clustering with $\boldsymbol{U}$.

Therefore, in the proposed algorithm, the Gaussian weighted partition matrix $\boldsymbol{U}_{\boldsymbol{G}}$ along with the conditional constraint $f_{k}$, shown in (13), is exploited instead of (11) and a new center set $\boldsymbol{y}_{\boldsymbol{i}}$ is derived by (14). The resulting estimation accuracy is increased even with low SNRs and it is demonstrated in the next section.

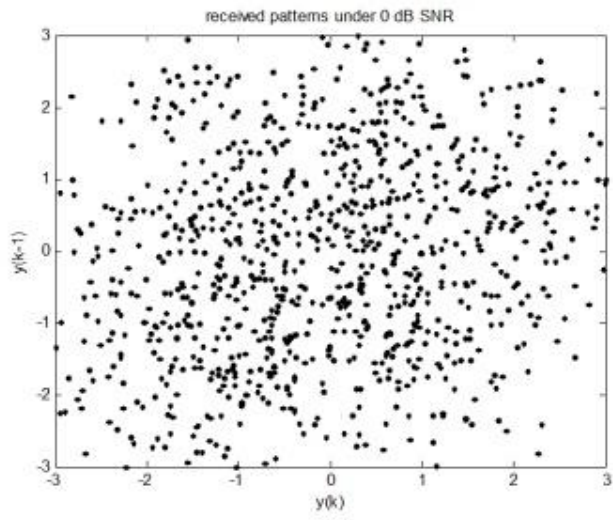

(a) received patterns under $0 \mathrm{~dB}$ SNR for the nonlinear channel in Table 1
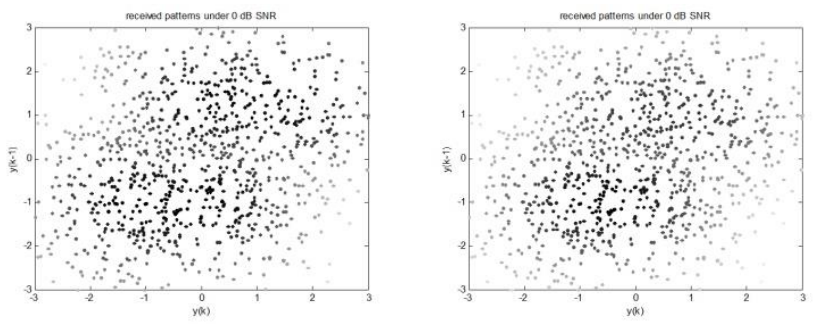

(b) received patterns displayed by $f_{k}: 1$ (black) $\leftrightarrow 0$ (white) (left: with $\boldsymbol{U}$, right: with $\boldsymbol{U}_{G}$ )
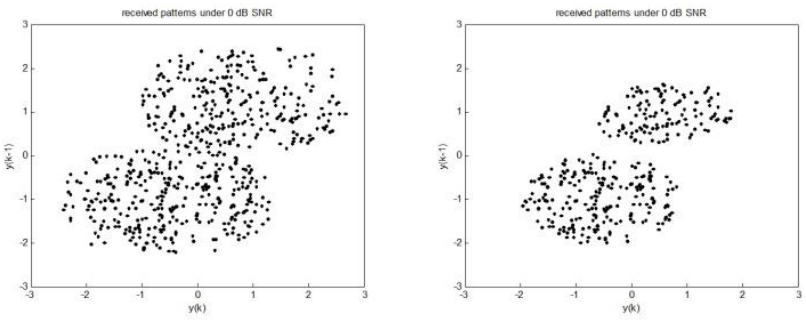

(c) received patterns only for $f_{k}>0.7$ (left: with $\boldsymbol{U}$, right: with $\boldsymbol{U}_{G}$ )
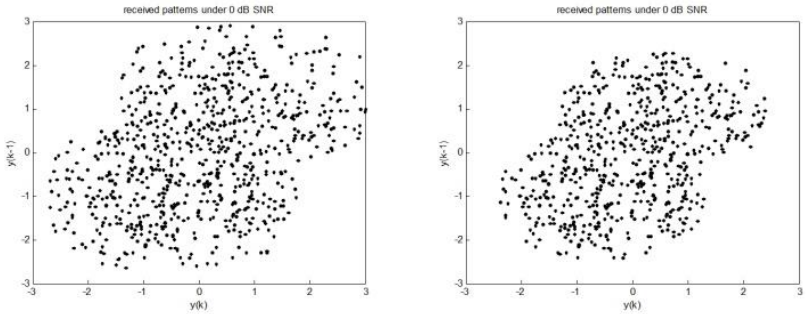

(d) received patterns only for $f_{k}>0.5$ (left: with $\boldsymbol{U}$, right: with $\boldsymbol{U}_{\boldsymbol{G}}$ )
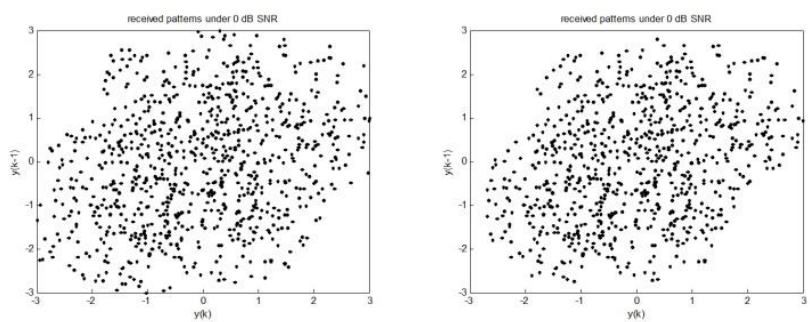

(e) received patterns only for $f_{k}>0.3$ (left: with $\boldsymbol{U}$, right: with $\boldsymbol{U}_{\boldsymbol{G}}$ )

Fig. 3. Received patterns under $0 \mathrm{~dB}$ SNR for the nonlinear channel in Table 1 and patterns displayed by their conditional constraint $f_{k}$ (left: clustering with $\boldsymbol{U}$, right: with $\boldsymbol{U}_{G}$ ).

\section{SimUlation RESUlTS AND COMPARISONS}

In order to demonstrate the performance of the proposed CFCM with $\boldsymbol{U}_{\boldsymbol{G}}$ in search of the optimal channel states for blind channel equalization, the following simulations are carried out and compared. As mentioned in the introduction section, the performance of CFCM in [16] was superior to the previously developed GA based [13][14] and conventional FCM based [15] approaches in terms of speed and accuracy. Those algorithms also estimate the optimal channel states of an unknown channel to solve the blind equalization problem. Therefore the comparison for the effectiveness of the proposed method focuses on the CFCM in [16]. In the experiments, three channels including a linear model are evaluated. Channel 1 and 2 shown in Table 1 stand for each of nonlinear and linear models respectively, with the channel order $p=1$, and Channel 3 discussed in Section 3 concerns a nonlinear model with the channel order $p=2$ as presented in [16] and [21]. The first two channels were also often discussed in [13]-[16]. The detailed description of the channels is presented below.

Channel 1 (nonlinear): $H(z)=0.5+1.0 z^{-1}$,

$$
D_{1}=1, D_{2}=0, D_{3}=-0.9, D_{4}=0, \text { and } d=1
$$

Channel 2 (linear): $H(z)=0.5+1.0 z^{-1}$,

$$
D_{1}=1, D_{2}=0, D_{3}=0, D_{4}=0, \text { and } d=1
$$

Channel 3 (nonlinear): $H(z)=0.3482+0.8704 z^{-1}+0.3482 z^{-2}$

$$
D_{1}=1, D_{2}=0.2, D_{3}=0, D_{4}=0, \text { and } d=1
$$


In the experiments, 10 independent simulations for each of three channels with five different noise levels $(\mathrm{SNR}=0,2.5,5$, 7.5 , and $10 \mathrm{~dB}$ ) were performed with 1,000 randomly generated transmitted symbols $(L=1000)$. Afterwards, the obtained results were averaged. The proposed CFCM with $\boldsymbol{U}_{\boldsymbol{G}}$ and the ordinary CFCM with $\boldsymbol{U}$ have been implemented in a batch mode to facilitate a comparative analysis. In addition, both algorithms are evaluated with the use of the same parameters shown in Table 2, and these are fixed for all experiments.

The choice of the specific parameter values is not critical to the performance of both algorithms. For the evaluation purpose, the normalized root mean squared errors (NRMSE) is determined in the form

$$
\mathrm{NRMSE}=\frac{1}{\|\boldsymbol{a}\|} \sqrt{\frac{1}{N} \sum_{i=1}^{N}\left\|\boldsymbol{a}-\hat{\boldsymbol{a}}_{\boldsymbol{i}}\right\|^{2}}
$$

where $\boldsymbol{a}$ is the data set of optimal channel output states, $\hat{\boldsymbol{a}}_{\boldsymbol{i}}$ is the data set of estimated channel output states in the $i^{\text {th }}$ simulation, and $N$ is the total number of independent simulations $(N=10)$.

TABLE II. PARAMETERS USED IN SIMULATIONS

\begin{tabular}{|l|c|c|}
\hline & $\begin{array}{c}\text { CFCM } \\
\text { with } \boldsymbol{U}\end{array}$ & $\begin{array}{c}\text { Proposed CFCM } \\
\text { with } \boldsymbol{U}_{\boldsymbol{G}}\end{array}$ \\
\hline Maximum number of iteration & 100 & 100 \\
\hline Threshold for $F F$ variation & $10^{-3}$ & $10^{-3}$ \\
\hline Exponent for partition matrix $\boldsymbol{U}$ & 2 & 1 \\
\hline Random initial channel states & {$[-0.50 .5]$} & {$[-0.50 .5]$} \\
\hline
\end{tabular}

The values of NRMSEs after 10 independent simulations for each of three channels are averaged and illustrated in Fig. 4. The proposed CFCM with $\boldsymbol{U}_{\boldsymbol{G}}$ comes with lower NRMSE for all three channels, and the performance differences are more severe in higher noise levels. As mentioned in the last part of Section 5 with Fig. 3, the clustering procedure with the Gaussian weighted partition matrix $\boldsymbol{U}_{\boldsymbol{G}}$ in the proposed modification makes it possible that, the closer located patterns near the optimal states have relatively higher values of $f_{k}$ than by clustering with $\boldsymbol{U}$ in the ordinary CFCM. Consequently, those patterns with higher $f_{k}$ are more influential in the clustering process.

This effectiveness of Gaussian weighted partition matrix $\boldsymbol{U}_{\boldsymbol{G}}$ is more critical in case of lower SNR because the type of corrupted noise in the channel is AWGN. Therefore the proposed CFCM with $\boldsymbol{U}_{\boldsymbol{G}}$ is highly effective to find the optimal channel states when the received patterns are heavily corrupted by AWGN.

A sample of 1,000 received symbols under 0dB SNR for Channel 3 and its desired channel states constructed from the estimated channel output states by the proposed and the ordinary CFCM are illustrated in Fig. 5.

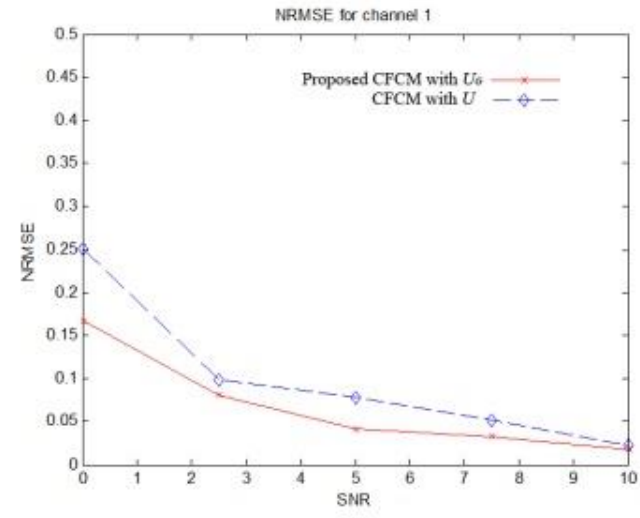

(a ) for channel 1

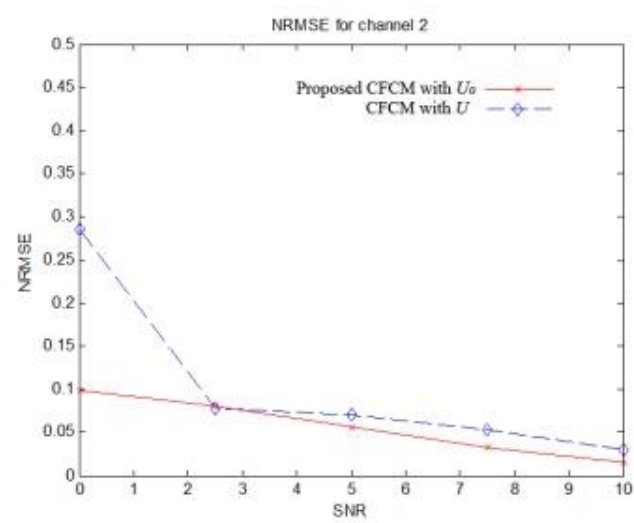

(b) for channel 2

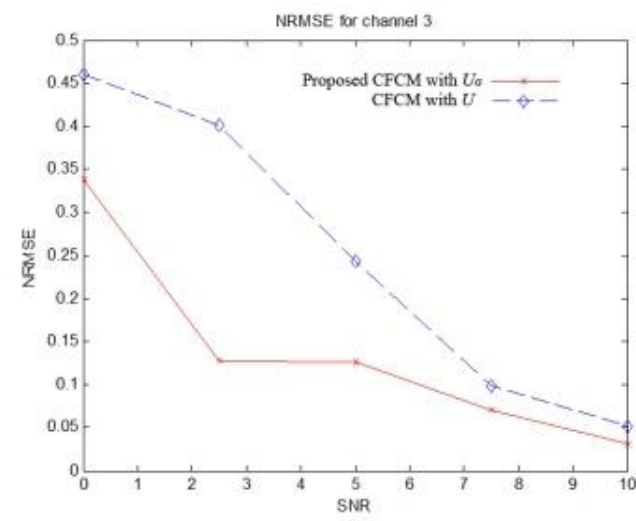

(c) for channel 3

Fig. 4. NRMSEs by the proposed CFCM with $\boldsymbol{U}_{\boldsymbol{G}}$ and by the ordinary CFCM with $\boldsymbol{U}$.

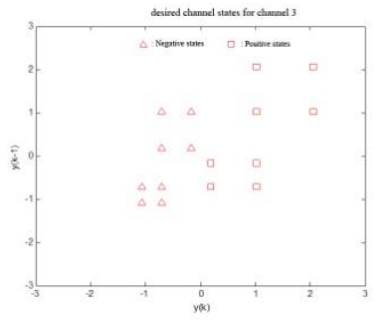

(a) optimal channel states for channel 3 (b) received patterns under $0 \mathrm{~dB}$ SNR

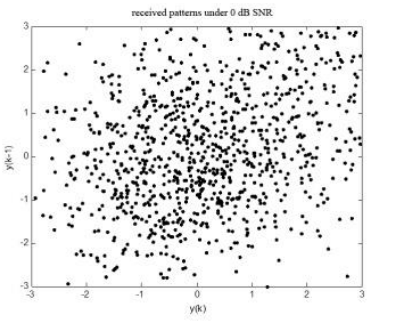




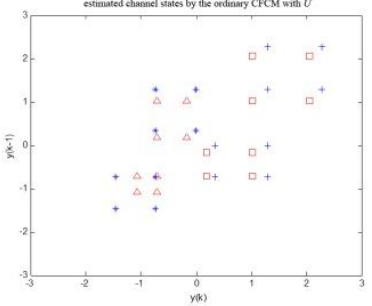

(c) estimated states by CFCM with $\boldsymbol{U}$

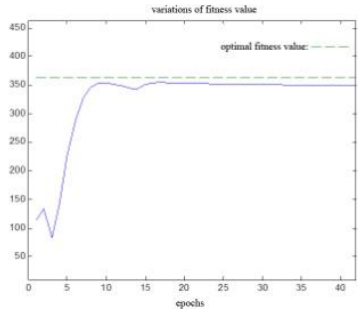

(e) fitness variations by CFCM with $\boldsymbol{U}$ (f) fitness variations by CFCM with $\boldsymbol{U}_{\boldsymbol{G}}$

Fig. 5. A sample of received symbols under OdB SNR for Channel 3 and its sixteen desired channel states estimated by the proposed CFCM with $\boldsymbol{U}_{\boldsymbol{G}}$ and the ordinary CFCM with $\boldsymbol{U}$.

Because of the use of $\boldsymbol{U}_{\boldsymbol{G}}$, the proposed CFCM produces more accurate channel states from the noise-corrupted received patterns as shown in Fig. 5(d), and its fitness value by (10) during the clustering procedure approaches the optimal fitness value more closely as in Fig. 5(f). In addition, the relative search time (RST) of the proposed CFCM with $\boldsymbol{U}_{\boldsymbol{G}}$ is evaluated. It is calculated by (16) and included in Table 4.

$R S T=\frac{\text { search time of CFCM with } U_{G}-\text { search time of CFCM with } U}{\text { search time of CFCM with } U}$

As shown in Table 3, the values of RST for all three channels with different noise levels are almost zero, which means the search time difference between two algorithms is not significant where the proposed CFCM provides much better performance in terms of NRMSE. Additionally, some of RST for Channel 3, especially with low SNRs, are negative (faster search time for the proposed CFCM). It is caused by using the $\boldsymbol{U}_{\boldsymbol{G}}$ in the clustering procedure, which reduces the number of convergence epochs in heavy noise circumstances.

TABLE III. Relative Search Time (RST) For All Three Channels WITH DIFFERENT NOISE LEVELS

\begin{tabular}{|c|c|c|}
\hline Channel & SNR & RST \\
\hline Channel 1 & $0.0 \mathrm{~dB}$ & 0.5588 \\
\cline { 2 - 3 } & $2.5 \mathrm{~dB}$ & 0 \\
\cline { 2 - 3 } & $5.0 \mathrm{~dB}$ & 0.0690 \\
\cline { 2 - 3 } & $7.5 \mathrm{~dB}$ & -0.1429 \\
\cline { 2 - 3 } & $10 \mathrm{~dB}$ & -0.1923 \\
\hline Channel 2 & $0.0 \mathrm{~dB}$ & 0.4653 \\
\cline { 2 - 3 } & $2.5 \mathrm{~dB}$ & 0.2 \\
\cline { 2 - 3 } & $5.0 \mathrm{~dB}$ & 0 \\
\cline { 2 - 3 } & $7.5 \mathrm{~dB}$ & -0.1724 \\
\cline { 2 - 3 } & $10 \mathrm{~dB}$ & -0.1154 \\
\hline Channel 3 & $0.0 \mathrm{~dB}$ & 0.2700 \\
\cline { 2 - 3 } & $2.5 \mathrm{~dB}$ & -0.4061 \\
\cline { 2 - 3 } & $5.0 \mathrm{~dB}$ & -0.2829 \\
\cline { 2 - 3 } & $7.5 \mathrm{~dB}$ & -0.4291 \\
\cline { 2 - 3 } & $10 \mathrm{~dB}$ & -0.2098 \\
\hline
\end{tabular}

Finally, the bit error rates (BER) with the optimal and the estimated channel states are evaluated by using the Bayesian equalizer and they are summarized in Table 4. Even though the BER with the estimated channel states realized by the proposed CFCM with $\boldsymbol{U}_{\boldsymbol{G}}$ is close enough to the one with the optimal channel states for all three channels, its performance does not dominate in terms of BER as much as it does in terms of NRMSE. Furthermore, especially for low SNRs, the BERs even with the optimal states are also relatively high. It is caused by the fact that the decision function of Bayesian equalizer shown in (7) is easily affected by heavy noise (high noise variance $\sigma_{e}^{2}$ ) even though the desired channel states can be estimated with high accuracy by using the proposed algorithm. For this reason, several nonlinear equalization techniques such as fuzzy or neural network implementations of Bayesian equalizer should be considered to improve the BER in next study.

TABLE IV. AVERAGED BER(\%) (No. OF ERRORS/No. OF TRANSMITTED SYMBOLS).

\begin{tabular}{|c|c|c|c|c|}
\hline \multirow{3}{*}{ Channel } & SNR & $\begin{array}{c}\text { with optimal } \\
\text { states }\end{array}$ & $\begin{array}{c}\text { Ordinary } \\
\text { CFCM } \\
\text { with } \boldsymbol{U}\end{array}$ & $\begin{array}{c}\text { Proposed } \\
\text { CFCM } \\
\text { with } \boldsymbol{U}_{\boldsymbol{G}}\end{array}$ \\
\hline Channel 1 & $0.0 \mathrm{~dB}$ & 19.8 & 21.6 & 20.1 \\
\cline { 2 - 5 } & $2.5 \mathrm{~dB}$ & 15.3 & 15.4 & 15.4 \\
\cline { 2 - 5 } & $5.0 \mathrm{~dB}$ & 10.7 & 10.6 & 10.6 \\
\cline { 2 - 5 } & $7.5 \mathrm{~dB}$ & 6.69 & 6.81 & 6.80 \\
\cline { 2 - 5 } & $10 \mathrm{~dB}$ & 2.77 & 2.79 & 2.75 \\
\hline Channel 2 & $0.0 \mathrm{~dB}$ & 19.3 & 21.6 & 19.2 \\
\cline { 2 - 5 } & $2.5 \mathrm{~dB}$ & 13.6 & 13.7 & 13.7 \\
\cline { 2 - 5 } & $5.0 \mathrm{~dB}$ & 8.95 & 9.17 & 9.08 \\
\cline { 2 - 5 } & $7.5 \mathrm{~dB}$ & 4.52 & 4.57 & 4.57 \\
\cline { 2 - 5 } & $10 \mathrm{~dB}$ & 1.79 & 1.76 & 1.76 \\
\hline Channel 3 & $0.0 \mathrm{~dB}$ & 22.1 & 23.0 & 22.5 \\
\cline { 2 - 5 } & $2.5 \mathrm{~dB}$ & 16.1 & 16.9 & 16.5 \\
\cline { 2 - 5 } & $5.0 \mathrm{~dB}$ & 11.7 & 12.6 & 11.8 \\
\cline { 2 - 5 } & $7.5 \mathrm{~dB}$ & 7.94 & 8.22 & 7.88 \\
\cline { 2 - 5 } & $10 \mathrm{~dB}$ & 4.89 & 5.28 & 4.97 \\
\hline
\end{tabular}

\section{CONCLUSIONS}

The determination of an unknown channel states only from received patterns is critical in blind linear/nonlinear channel equalization problems. In this paper, for the estimation of desired channel states of an unknown digital channel under severe noise-corrupted communication environments, a modification of CFCM with Gaussian weighted partition matrix is presented and successfully evaluated with both of linear and nonlinear channels. Especially even when the received symbols are significantly corrupted by a heavy AWGN, it can estimate the channel output states with relatively high accuracy and substantial speed. Therefore, in the presence of heavy AWGN, the Bayesian equalizer with the proposed CFCM can be a possible solution for blind channel equalization. In future works, the evaluation of this method with higher order channels is included. In addition, as mentioned at the end of the last section, a further study on the implementation methods of Bayesian equalizer should be included to improve the BER under the presence of severe noise.

\section{REFERENCES}

[1] J. G. Proakis, Digital Communications, Fourth edition, McGraw-Hill, New York, 2001 
[2] H. Gazzah and K. A. Meraim, "Blind ZF equalization with controlled delay robust to order over estimation", Signal Processing, vol.83, pp.1505-1518, 2003.

[3] Yun Ye and Saman S. Abeysekera, "Efficient blind estimation and equalization of non-minimum phase communication channels via the use of a zero forcing equalizer", Signal Processing, vol. 86, pp.1019-1034, 2006.

[4] J.R. Barry, E. A. Lee and D. G. Messerschmitt, Digital Communication, $3^{\text {rd }}$ ed. Norwell, MA: Kluwer, 2004.

[5] D. Erdogmus, D. Rende, J.C. Principe and T.F. Wong, "Nonlinear channel equalization using multilayer perceptrons with information theoretic criterion", Proc. of IEEE workshop Neural Networks and Signal Processing, pp. 443-451, MA, U.S.A., 2001.

[6] N. Xie and H. Leung, "Blind equalization using a predictive radial basis function neural network," IEEE Transactions on Neural Networks, vol. 16, no. 3, pp. 709-720, 2005.

[7] M. Mimura and T. Furukawa, "A recurrent RBF network for non-linear channel," Proc. of 2001 IEEE ICASSP, vol. 2, pp.1297-1300, UT., U.S.A., 2001.

[8] G. A. Barreto and L. G. M. Souza, "Adaptive filtering with the selforganizing map: A performance comparison," Neural Networks, vol. 19, no. 6, pp. 785-798, 2006.

[9] S. Han, "Blind Equalization of Linear/Nonlinear Channels by SOM", International Journal of Informaation Technology and Network Application, vol. 2, no. 3, pp. 19-27, 2012.

[10] A.K. Pradhan, S.K. Meher and A. Routray, "Communication channel equalization using wavelet network," Digital Signal Processing, vol. 16, no. 4, pp. 445-452, July, 2006.

[11] B. Mitchinson and R. F. Harrison, "Digital communications channel equalization using the kernel adaline," IEEE Transactions on Communications, vol. 50, no. 4, pp. 571-576, 2002.
[12] I. Santamaria, C. Pantaleon, L. Vielva and J. Ibanez, "Blind Equalization of Constant Modulus Signals Using Support Vector Machines", IEEE Trans. Signal Processing, vol. 52, pp.1773-1782, 2004.

[13] H. Lin and K. Yamashita, "Hybrid simplex genetic algorithm for blind equalization using RBF networks", Mathematics and Computers in Simulation, vol. 59, pp.293-304, 2002

[14] S. Han, W. Pedrycz and C. Han, "Nonlinear Channel Blind Equalization Using Hybrid Genetic Algorithm with Simulated Annealing", Mathematical and Computer Modeling, vol. 41, pp.697-709, 2005.

[15] S. Han, I. Lee and W. Pedrycz, "Modified fuzzy c-means and Bayesian equalizer for nonlinear blind channel", Applied Soft Computing, vol. 9, pp.1090-1096, 2009.

[16] S. Han, S. Park and W. Pedrycz "Conditional fuzzy clustering for blind channel equalization”, Applied Soft Computing, vol. 11, pp.2777-2786, 2011.

[17] W. Pedrycz, "Conditional Fuzzy Clustering in the Design of Radial Basis Function Neural Networks", IEEE Trans. Neural Networks, vol. 9, pp.601-612, 1998.

[18] K. Yoon, K. Kwak and S. Kim, "Nonlinear channel equalization using fuzzy clustering adaptive neuro-fuzzy filter", Journal of Korea Electronics Engineers Society, vol. 38, pp.35-41, 2001.

[19] S. Chen, B. Mulgrew and S. McLaughlin, "Adaptive Bayesian equalizer with decision feedback", IEEE Trans. Signal Processing, vol. 41, pp.2918-2927, 1993.

[20] H. Lin and K. Yamashita, "Blind equalization using parallel Bayesian decision feedback equalizer", Mathematics and Computers in Simulation, vol. 56, pp.247-257, 2001

[21] S.K. Patra and B. Mulgrew, "Fuzzy techniques for adaptive nonlinear equalization", Signal Processing, vol. 80, pp.985-1000, 2000. 